\title{
Overexpression of p28GANK accelerates the metastasis of oesophageal squamous cell carcinoma
}

\author{
SHANHONG TANG ${ }^{1,2^{*}}$, JIANPING QIN $^{1 *}$, WEIHUI LIU ${ }^{3 *}$, XIUSHAN ZHENG ${ }^{4 *}$, XIAOLING WU ${ }^{1}$, \\ HUI XU ${ }^{1}$, LIJUAN QIAO ${ }^{1}$, QUANSHUI FAN ${ }^{2}$, WEIZHENG ZENG ${ }^{1}$ and MINGDE JIANG ${ }^{1}$ \\ ${ }^{1}$ Department of Digestion, General Hospital of Chengdu Military Command; \\ ${ }^{2}$ Prevention and Control Centre of Disease of Chengdu Military Command; Departments of ${ }^{3}$ General Surgery and ${ }^{4}$ Thoracic Surgery, \\ General Hospital of Chengdu Military Command, Chengdu, Sichuan 610083, P.R. China
}

Received March 5, 2014; Accepted October 24, 2014

DOI: $10.3892 / \mathrm{mmr} .2015 .3286$

\begin{abstract}
The present study aimed to evaluate the effects of p28GANK expression on the metastasis of oesophageal squamous cell carcinoma (ESCC) tissues and to investigate its roles in the metastasis of highly invasive and non-invasive ESCC cell lines. Quantitative polymerase chain reaction (qPCR) and immunohistochemical analyses were performed to assess p28GANK mRNA and protein expression in ESCC tissues and to analyse its significance in ESCC metastasis. qPCR and western blot analyses were used to detect p28GANK mRNA and protein expression in highly invasive and non-invasive cell lines. Subsequently, lentivirus-mediated p28GANK short interfering RNA (siRNA) was transfected into highly invasive ESCC cells, and Transwell assays were performed to analyse the effects of p28GANK knockdown on their migration and invasion. The mean expression levels of p28GANK mRNA in the ESCC tissues of patients with metastasis were significantly higher than those in the ESCC specimens from patients without metastasis. p28GANK expression in ESCC tissues was correlated with T-stage, lymph node metastasis and lymphatic invasion. The mRNA and protein expression levels of p28GANK were significantly higher in highly invasive cell lines compared with those of matched, non-invasive cell lines. Lentivirus-mediated siRNA knockdown of p28GANK markedly decreased p28GANK expression in EC109-P and EC9706-P cells and supressed the metastasis of ESCC cells in vitro. In conclusion, p28GANK
\end{abstract}

Correspondence to: Professor Weizheng Zeng or Professor Mingde Jiang, Department of Digestion, General Hospital of Chengdu Military Command, 72 Rongdu Road, Chengdu, Sichuan 610083, P.R. China

E-mail: zengweizheng@163.com

E-mail: 15928956390@163.com

*Contributed equally

Key words: oesophageal squamous cell carcinoma, p28GANK, invasiveness, metastasis expression was increased in metastatic ESCC tissues and cells, and p28GANK knockdown decreased the metastatic ability of ESCC cells. These results suggested that p28GANK may be a potential therapeutic marker for ESCC metastasis.

\section{Introduction}

Oesophageal squamous cell carcinoma (ESCC) is one of the most common types of malignant tumour, accounting for $\sim 80 \%$ of patients with cancer from developing countries, including Northern China $(1,2)$. The clinical outcome and prognosis of patients are poor due to the highly invasive and metastatic nature of the disease. In previous studies by our group, Twist (3), short chain dehydrogenase/reductase family 9C, member 7 (4) and bone morphogenetic protein 7 (5) were found to have significant roles in the metastasis of ESCC. However, the precise mechanisms underlying the metastasis of ESCC remain to be elucidated. Therefore, the identification of potential markers for the diagnosis and treatment of ESCC metastasis is critical.

p28GANK, which is also known as PSMD10 or gankyrin, is the product of the p28 gene and a subunit of the regulatory complex of the human $26 \mathrm{~S}$ proteosome, with seven ankyrin repeats. p28GANK regulates the malignant growth of cancer cells by activating murine double minute 2 , leading to the proteasomal degradation of the tumour suppressor gene p53. A previous study by our group revealed that overexpression of p28GANK accelerated the malignant progression of human colorectal and pancreatic cancers $(6,7)$. The overexpression of p28GANK has also been shown to accelerate the invasive ability and metastasis of hepatocellular carcinoma (8), breast cancer (9), oral cancer (10) and glioma (11). In addition, p28GANK was demonstrated to be essential for hypoxia-enhanced metastatic potential in breast cancer cells (12). The overexpression p28GANK confers multidrug resistance to liver and gastric cancer cells (13). In ESCC, p28GANK overexpression was associated with poor prognosis and promoted tumour progression (14). However, the specific role of p28GANK in ESCC metastasis remains to be elucidated.

In the present study, the significance of p28GANK expression in ESCC metastasis was evaluated using quantitative 
polymerase chain reaction (qPCR) and immunohistochemical analyses. Additionally, western blot analysis was performed to assess p28GANK expression in highly invasive and non-invasive ESCC cells. Finally, in order to investigate the role of p28GANK in ESCC metastasis, lentivirus-mediated short interfering RNA (siRNA), targeting p28GANK was transfected into highly invasive ESCC cells.

\section{Materials and methods}

Clinical specimens. For immunohistochemical staining, paraffin-embedded ESCC tissues from 112 patients (84 males, 28 females), aged 36-72 years (mean, 57.2 years), were obtained between 2008 and 2010 at the General Hospital of Chengdu Military Command (Chengdu, China). For qPCR analysis, 52 fresh ESCC tissue samples were obtained, of which 26 were from patients with metastasis and 26 were from patients without metastasis. All samples were immediately placed in liquid nitrogen, in which they were stored prior to use. All patients received ESCC resections between January 2010 and January 2013 in the Department of Chest Surgery, General Hospital of Chengdu Military Command, Chengdu, China. These patients did not undergo chemotherapy or radiotherapy prior to surgical ablations and written consent for the use of ESCC tissues was obtained from all patients. The present study was authorised by the Ethical Committee of the General Hospital of Chengdu Military Command (Chengdu, China).

ESCC cell lines. ESCC cell lines EC109 and EC9706 were purchased from the Chinese Academy of Medical Science (Beijing, China) and maintained in RPMI-1640 medium (Gibco-BRL, Invitrogen Life Technologies, Carlsbad, CA, USA) supplemented with $10 \%$ foetal bovine serum (Gibco-BRL) at $37^{\circ} \mathrm{C}$ in humidified air containing $5 \% \mathrm{CO}_{2}$. Highly and non-invasive ESCC sub-lines were constructed using repeated Transwell assays as described previously (4). Following a ten-round selection and expansion, the highly invasive sub-lines were established and named EC9706-P and EC109-P, and the non-invasive sub-lines were named EC109-N and EC9706-N.

qPCR. A qPCR assay was performed to detect p28GANK mRNA expression in ESCC tissues and highly invasive and non-invasive cell lines, and the results were calculated as previously described (15). The primer sequences used were as follows: p28GANK forward, 5'-TCTTCAAGCCATCCTGTGTG-3' and reverse, 5'-TGGTGATGTTGGACT CCTCA-3'; $\beta$-actin (internal control) forward, 5'-ATGATATCGCCGCGCTCGTC-3' and reverse, 5'-CGCTCGGTGAGG ATCTTCA-3' (Huada Gene Biotechnology Co., Ltd., Shenzheng, Guangdong, China).

Immunohistochemical staining. Immunohistochemical staining was performed as previously described (8). Briefly, the paraffin-embedded tissues were deparaffinised, rehydrated, quenched of endogenous peroxidase activity and blocked with goat serum (Zhongshan Golden Bridge Biotechnology Co., Ltd., Beijing, China). The tissues were incubated with rabbit anti-gankyrin antibody (1:50; Santa
Cruz Biotechnology, Inc., Dallas, TX, USA) overnight at $4^{\circ} \mathrm{C}$. Following three washes with phosphate-buffered saline Tween 20, the tissues were incubated with biotinylated goat anti-rabbit immunoglobulin $\mathrm{G}$ ( $\mathrm{IgG}$ )/horseradish peroxidase (HRP) $(1 ; 2,000$; Zhongshan Golden Bridge Biotechnology Co., Ltd.) for $50 \mathrm{~min}$ at room temperature. 3,3-diaminobenzidine tetrahydrochloride was used for visualisation. The stained cells were counted, and the results were scored based on the proportion of stained cells $(0 \%, 0 ; 1-33 \%, 1 ; 34-66 \%$, 2 and $67-100 \%, 3$ ) and intensity (achromatic, 0; amber, 1; yellow, 2 and brown, 3) of staining. Immunoreactivity was determined by the combined scores as follows: Negative (-), 0-2; positive (+), 3-6. Ten representative fields for each sample were counted, using an SZ51 microscope (Olympus Corporation, Tokyo, Japan).

Western blot analysis. Western blot analyses were performed as previously described (8). Total protein lysates were separated by $12 \%$ SDS-PAGE and transferred onto nitrocellulose membranes. The membranes were blocked with $10 \%$ fat-free milk and incubated with rabbit polyclonal anti-p28GANK (1:100; Santa Cruz Biotechnology, Inc.) and mouse monoclonal anti- $\beta$-actin (1:3,000; Sigma-Aldrich, St. Louis, MO, USA). Following incubation with monoclonal HRP-coupled goat anti-mouse IgG (1:2,000; BiosPacific, Inc., Emeryville, CA, USA) and goat anti-rabbit (1:2,000; BiosPacific, Inc.) secondary antibodies and enhanced chemiluminescence solution (ECL-kit; Thermo Fisher Scientific, Waltham, MA, USA) was used to visualise the signals. The protein expression results were based on the strip area of the target proteins, relative to the controls.

Lentivirus-mediated siRNA transfection. The p28GANK siRNA (5'-CTGACCAGGACAGCAGAAC-3') was sub-cloned into the PGC-lentiviral system with enhanced green fluorescent protein (EGFP). The siRNA and control lentiviruses generated were subsequently transfected into highly invasive EC9706-P and EC109-P cells, and the transfected cells were named Con-EC9706-P, Si-EC9706-P, Con-EC109-P and Si-EC109-P, respectively.

Transwell assays. Transwell assays, including cell migration and invasion assays, were performed as previously described (4). Once the cells on the upper membrane surface were removed, the number of cells on the lower surface was counted under a $\times 200$ visual field using an inverted microscope. Five representative fields were counted for each cell line using an SZ51 microscope (Olympus Corporation) and the results presented are the mean of five representative experiments.

Statistical analysis. The results were analysed using SPSS 17.0 software (SPSS, Inc., Chicago, IL, USA), and $\mathrm{P}<0.05$ was considered to indicate a statistically significant difference between values. Student's t-test was used to analyse differences in mRNA expression. The Kruskal-Wallis test was performed to analyse the potential correlations between p28GANK expression and the clinical parameters of patients with ESCC. The one-way analysis of variance test was used to analyse differences in the Transwell assays. 
Table I. Significance of p28GANK expression in oesophageal squamous cell carcinoma tissues.

\begin{tabular}{|c|c|c|c|c|c|c|}
\hline \multirow[b]{2}{*}{ Factor } & \multirow[b]{2}{*}{$\mathrm{n}$} & \multicolumn{4}{|c|}{ p28GANK expression } & \multirow[b]{2}{*}{ P-value } \\
\hline & & - & \pm & + & ++ & \\
\hline Gender & & & & & & 0.51 \\
\hline Female & 28 & 6 & 9 & 11 & 2 & \\
\hline Male & 84 & 17 & 22 & 35 & 10 & \\
\hline Age (years) & & & & & & 0.58 \\
\hline$\leq 57$ & 56 & 12 & 17 & 21 & 6 & \\
\hline$>57$ & 56 & 11 & 14 & 25 & 6 & \\
\hline T stage & & & & & & 0.01 \\
\hline I & 12 & 5 & 5 & 1 & 1 & \\
\hline II & 46 & 11 & 14 & 17 & 4 & \\
\hline III & 48 & 7 & 12 & 23 & 6 & \\
\hline IV & 6 & 0 & 0 & 5 & 1 & \\
\hline Metastasis & & & & & & $<0.01$ \\
\hline N0 & 50 & 21 & 18 & 11 & 0 & \\
\hline N1 & 35 & 1 & 6 & 20 & 8 & \\
\hline N2 & 23 & 1 & 5 & 13 & 4 & \\
\hline N3 & 4 & 0 & 2 & 2 & 0 & \\
\hline Differentiation & & & & & & 0.08 \\
\hline Well & 1 & 5 & 9 & 3 & 18 & \\
\hline Moderate & 8 & 10 & 19 & 5 & 42 & \\
\hline Poor & 14 & 16 & 18 & 4 & 52 & \\
\hline Lymphatic invasion & & & & & & $<0.01$ \\
\hline Without & 55 & 18 & 21 & 15 & 1 & \\
\hline With & 57 & 5 & 10 & 31 & 11 & \\
\hline
\end{tabular}

$\mathbf{A}$
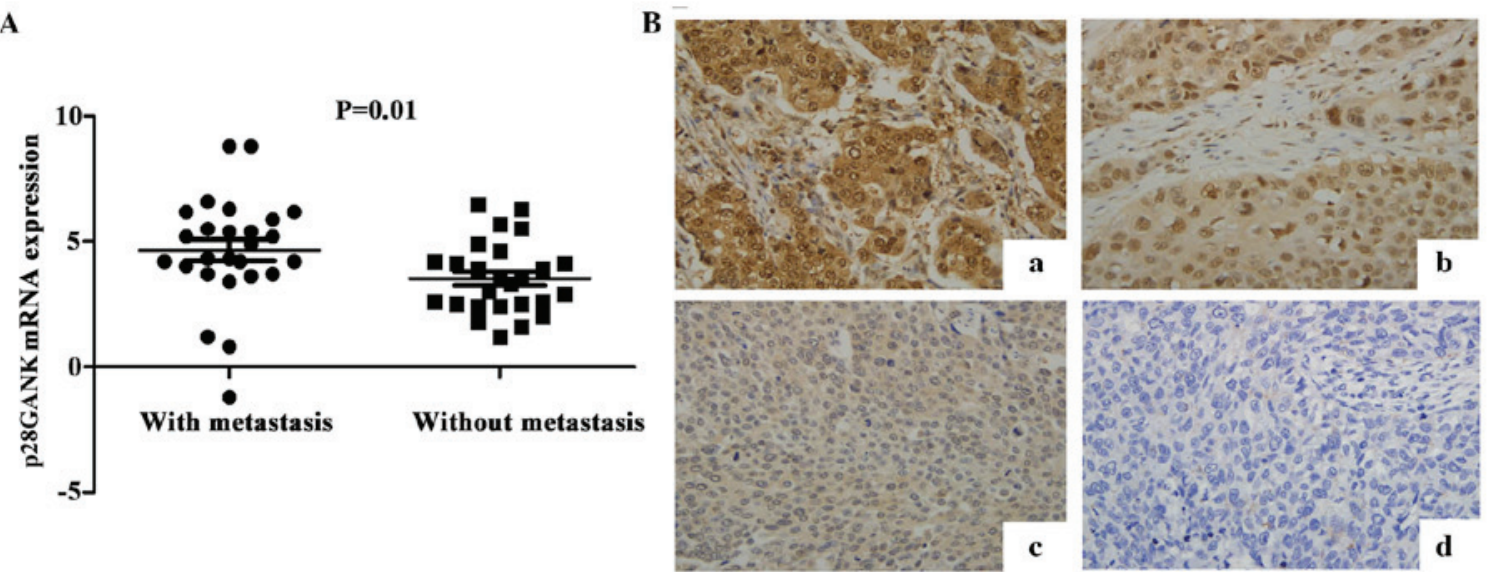

Figure 1. p28GANK expression in ESCC tissues with or without metastasis. (A) p28GANK mRNA expression levels were assessed by quantitative polymerase chain reaction analysis. Mean p28GANK expression levels were significantly higher in ESCC tissues from patients with metastasis compared with those from patients without metastasis $(\mathrm{P}=0.01)$. (B) Immunohistochemical staining was performed to detect p28GANK expression in ESCC tissues from 112 patients: (a) Strong positive staining (++) of p28GANK in ESCC tissues from patients with metastasis; (b) moderately positive staining (+) of p28GANK in ESCC tissues from patients with metastasis; (c) weakly positive staining ( \pm ) of p28GANK in ESCC tissues from patients without metastasis; (d) absence of staining (-) of p28GANK in ESCC tissues from patients without metastasis. Magnification, x200. ESCC, oesophageal squamous cell carcinoma; mRNA, messenger RNA.

\section{Results}

p28GANK is highly expressed in metastatic ESCC tissues. To investigate the significance of p28GANK expression in ESCC metastasis, the expression of p28GANK mRNA in 52 ESCC tissues was evaluated using qPCR analysis. The mean expression levels of p28GANK mRNA in ESCC tissues from patients with metastasis were significantly higher $(4.65 \pm 2.16)$ 

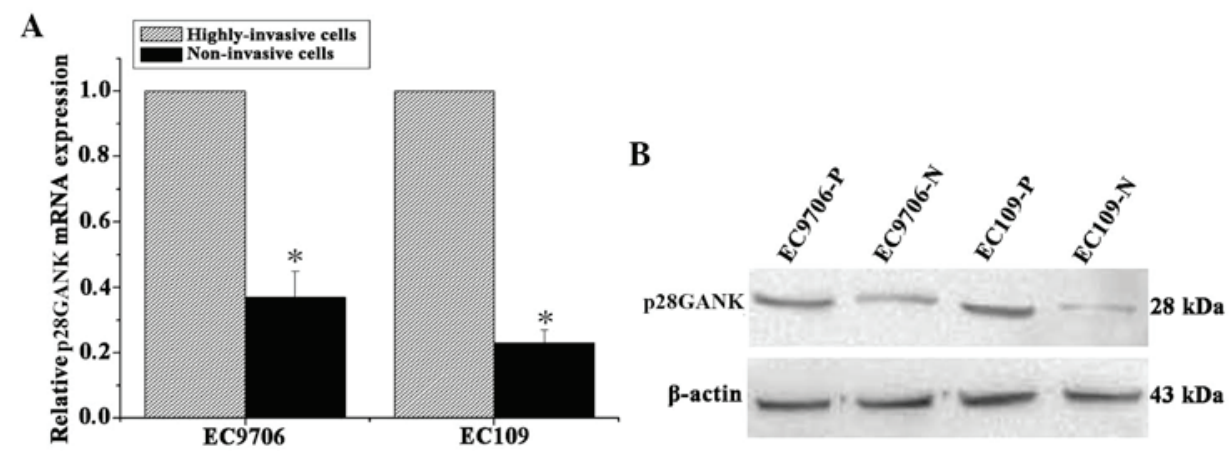

Figure 2. p28GANK expression in highly invasive and non-invasive oesophageal squamous cell carcinoma cell lines. (A) Quantitative polymerase chain reaction analysis indicated that p28GANK mRNA expression levels were significantly higher in highly invasive cells compared with those of matched, non-invasive cells; ${ }^{*} \mathrm{P}<0.05$ vs. matched, highly invasive cells. (B) Western blot analysis demonstrated that p28GANK expression was markedly higher in highly invasive cells compared with matched, non-invasive cells $(\mathrm{P}<0.01)$. mRNA, messenger RNA.
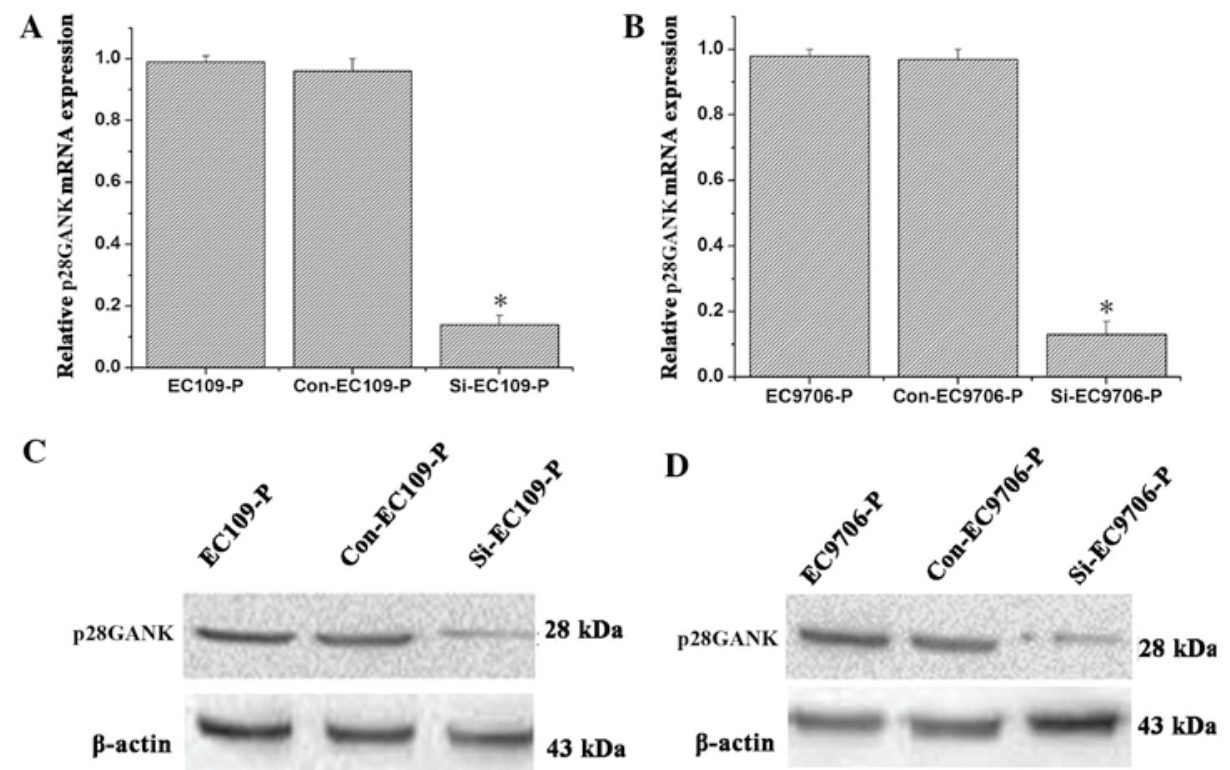

Figure 3. Lentivirus-mediated siRNA targeting p28GANK significantly inhibited p28GANK expression in highly invasive oesophageal squamous cell carcinoma cells. (A and B) Polymerase chain reaction analysis indicated that lentivirus-mediated siRNA knockdown markedly inhibited p28GANK mRNA expression levels in the EC109-P and EC9706-P cell lines. (C and D) Western blot analysis also indicated that p28GANK expression levels were markedly decreased in p28GANK siRNA-transfected cells compared with those of matched controls. "P $<0.05$ for Si-EC109-P or Si-EC9706-P vs. matched controls. siRNA, short interfering RNA; Con, control; Si, group transfected with siRNA.

than those of ESCC specimens from patients without metastasis $(3.52 \pm 1.43 ; \mathrm{P}=0.01 ;$ Fig. $1 \mathrm{~A})$.

p28GANK expression in ESCC tissues is correlated with T-stage, lymph node metastasis and lymphatic invasion. Immunohistochemical staining was performed to assess the expression of p28GANK protein in ESCC tissues from 112 patients. Positive p28GANK expression was observed in the cytoplasm and/or nucleus in ESCC tissues (Fig. 1B). The distribution of negative, weakly positive, moderately positive and strongly positive p28GANK expression were 20.5\% (23/112), 27.7\% (31/112), 41.1\% (46/112) and 10.7\% (12/112), respectively. Statistical analysis indicated that $\mathrm{p} 28 \mathrm{GANK}$ expression was correlated with ESCC T-stage $(\mathrm{P}=0.01)$, lymph node metastasis $(\mathrm{P}<0.01)$ and lymphatic invasion $(\mathrm{P}<0.01)$. However, there was no significant correlation between
p28GANK expression and patient age, gender or level of differentiation (Table I; P>0.05).

p28GANK expression is increased in highly invasive ESCC cell lines. The highly invasive and the non-invasive ESCC cell lines were constructed using repeated Transwell assays, as described in a previous study by our group (4). qPCR analysis revealed that p28GANK mRNA expression levels were significantly higher in the highly invasive ESCC cell lines, EC109-P and EC9706-P, compared with those in their matched, non-invasive cell lines, EC109-N and EC9706-N (Fig. 2A). Consistent with the results of qPCR analysis, western blot analysis indicated that the expression levels of p28GANK protein were markedly higher in the highly invasive cell lines compared with those in matched non-invasive cell lines (Fig. 2B). These results indicated that p28GANK expression 



Figure 4. p28GANK knockdown inhibited the migration and invasion of highly invasive oesophageal squamous cell carcinoma cells. (A and B) p28GANK knockdown decreased the migration and invasion of EC109-P cells. (C and D) p28GANK knockdown supressed the migration and invasion of EC9706-P cells. Magnification, $\mathrm{x} 400 .{ }^{*} \mathrm{P}<0.05$ vs. matched control. Con, control; Si, transfected with short interfering RNA.

levels were increased in highly invasive ESCC cells compared with those in non-invasive ESCC cells.

Decreased p28GANK expression suppresses the migration and invasion of highly invasive ESCC cells. Lentivirus-mediated siRNA, targeting p28GANK was used to evaluate the effects of p28GANK knockdown on the metastatic phenotypes of highly invasive ESCC cells. qPCR analysis demonstrated that lentivirus-mediated knockdown of p28GANK with siRNA markedly inhibited p28GANK mRNA expression in the EC109-P and EC9706-P cell lines (Fig. 3A and B). Consistent with the results of $\mathrm{qPCR}$ analysis, western blot analysis indicated that p28GANK expression levels were markedly decreased in p28GANK siRNA transfected cells compared with those in matched controls (Fig. 3C and D). These results revealed that the lentivirus-mediated siRNA targeting of p28GANK markedly decreased p28GANK expression in EC109-P and EC9706-P cells.

As shown in Fig. 4, Transwell assays revealed that the migration and invasion abilities of p28GANK-silenced cells were significantly lower than those of matched controls, indicating that p28GANK inhibition repressed ESCC cell metastasis in vitro.

\section{Discussion}

P28GANK is a regulator of the 26 S proteasome, which regulates the anti-apoptotic ability of cells by increasing the proteasome-mediated degradation of p53 and results in the alteration of p53-dependent gene expression (16). The overexpression of p28GANK upregulates the phosphorylation of retinoblastoma 1 (RB1) and downregulates it, via its RB1-binding motif. This promotes oncogenic transformation (17) and degradation of Oct4, and promotes the expansion of tumour-initiating cells in hepatocarcinogenesis (18). These studies revealed significant roles for p28GANK in the development of malignant human tumours. In addition, p28GANK was shown to increase the epithelial-mesenchymal transition and the motility/invasiveness of hepatocellular carcinoma cells, a process which is mediated by the activation of hypoxia-inducible factor $1 \alpha$ signalling and consequently, promotes TWIST1, vascular endothelial growth factor and metalloproteinase 2 expression (18). Increased p28GANK expression is correlated with poor prognosis and may have a significant role in ESCC tumour progression (14). Therefore, p28GANK may be a potentially important therapeutic target gene in ESCC (14). However, the significance and specific function of p28GANK in the metastasis of ESCC had remained to be elucidated.

In the present study, the significance of p28GANK mRNA expression in the metastasis of ESCC was evaluated. qPCR analysis indicated that the levels of p28GANK mRNA were significantly increased in metastatic ESCC tissues compared with those of non-metastatic ESCC tissues. Furthermore, the results of the immunohistochemical assay indicated that p28GANK expression was significantly correlated with T-stage, lymph node metastasis and lymphatic invasion. These findings were consistent with a previous study, which indicated that positive p28GANK expression was correlated with the extent of the primary tumour, lymph node metastasis and distant lymph node metastasis (14). In addition, the significance of p28GANK expression in the metastasis of ESCC was confirmed and it was demonstrated that p28GANK was able to serve as a biomarker for differentiating ESCC with or without metastatic potential.

In a previous study by our group, highly invasive and non-invasive cell subpopulations were isolated from established the ESCC cell lines, EC109 and EC9706-P, using the repeated 
Transwell approach described in studies investigating tumour metastasis $(19,20)$. The two pairs of highly invasive and non-invasive cell subpopulations that were obtained, were ideal models for studying ESCC metastasis (4). Therefore, p28GANK expression was assessed in the highly invasive and non-invasive cell subpopulations using qPCR and western blot analyses. Consistent with the results regarding p28GANK expression in ESCC tissues, mRNA and protein expression levels of p28GANK were markedly higher in the highly invasive cell lines, EC109-P and EC9706-P, compared with those of matched, non-invasive cell lines, CE109-N and EC9706-N. Furthermore, lentivirus-mediated siRNA knockdown of p28GANK markedly decreased the expression of p28GANK in EC109-P and EC9706-P cells, and Transwell assays revealed that the migration and invasion abilities of the p28GANK knockdown cells were significantly decreased. These results indicated that p28GANK inhibition suppressed the metastasis of ESCC cells in vitro. p28GANK promoted the hepatic metastasis of colorectal cancer by activating the interleukin- 8 signalling pathway (21) and controlled stem-cell behaviours by regulating the expression of stemness factors (22). p28GANK has significant roles in the metastasis of breast cancer by regulating Ras-related C3 botulinum toxin substrate 1 activity and may be a potential therapeutic target for breast cancer metastasis (23). The present study confirmed that p28GANK was also critical for the metastasis of human ESCC, although the precise mechanisms underlying these observations remain to be elucidated.

In conclusion, the present study demonstrated that the overexpression of $\mathrm{p} 28 \mathrm{GANK}$ promoted the metastasis of ESCC in tissues and cell lines and that p28GANK knockdown significantly inhibited the metastatic potential of ESCC. This suggested that p28GANK may function as a biomarker for differentiating ESCC with or without metastatic potential and may be a potential therapeutic marker for ESCC metastasis.

\section{Acknowledgements}

The present study was supported by a grant from the National Natural Science Foundations of China (grant no. 81401993, to Tang S).

\section{References}

1. Yu C, Chen K, Zheng H, et al: Overexpression of astrocyte elevated gene-1 (AEG-1) is associated with esophageal squamous cell carcinoma (ESCC) progression and pathogenesis. Carcinogenesis 30: 894-901, 2009.

2. Mohebbi M, Mahmoodi M, Wolfe R, et al: Geographical spread of gastrointestinal tract cancer incidence in the Caspian Sea region of Iran: spatial analysis of cancer registry data. BMC Cancer 8: 137, 2008.
3. Gong T, Xue Z, Tang S, et al: Nuclear expression of Twist promotes lymphatic metastasis in esophageal squamous cell carcinoma. Cancer Biol Ther 13: 606-613, 2012.

4. Tang S, Gao L, Bi Q, et al: SDR9C7 promotes lymph node metastases in patients with esophageal squamous cell carcinoma. PLoS One 8: e52184, 2013.

5. Xu G, Tang S, Yang J, et al: BMP7 expression in esophageal squamous cell carcinoma and its potential role in modulating metastasis. Dig Dis Sci 58: 1871-1879, 2013.

6. Meng Y, He L, Guo X, et al: Gankyrin promotes the proliferation of human pancreatic cancer. Cancer Lett 297: 9-17, 2010.

7. Tang S, Yang G, Meng Y, et al: Overexpression of a novel gene gankyrin correlates with the malignant phenotype of colorectal cancer. Cancer Biol Ther 9: 88-95, 2010.

8. Fu J, Chen Y, Cao J, et al: p28GANK overexpression accelerates hepatocellular carcinoma invasiveness and metastasis via phosphoinositol 3-kinase/AKT/hypoxia-inducible factor-1 $\alpha$ pathways Hepatology 53: 181-192, 2011.

9. Zhen C, Chen L, Zhao Q, et al: Gankyrin promotes breast cancer cell metastasis by regulating Racl activity. Oncogene 32: 3452-3460, 2013

10. Li J, Knobloch TJ, Kresty LA, et al: Gankyrin, a biomarker for epithelial carcinogenesis, is overexpressed in human oral cancer. Anticancer Res 31: 2683-2692, 2011.

11. Yang Y, Zhang C, Li L, et al: Up-regulated oncoprotein P28GANK correlates with proliferation and poor prognosis of human glioma. World J Surg Oncol 10: 169, 2012.

12. Gao L, Xie H, Dong L, et al: Gankyrin is essential for hypoxia enhanced metastatic potential in breast cancer cells. Mol Med Rep 9: 1032-1036, 2014.

13. Wang G, Rong J, Zhou Z and Duo J: Novel gene P28GANK confers multidrug resistance by modulating the expression of MDR-1, Bcl-2 and Bax in osteosarcoma cells. Mol Biol (Mosk) 44: 1010-1017, 2010.

14. Ortiz CM, Ito T, Tanaka E, et al: Gankyrin oncoprotein overexpression as a critical factor for tumor growth in human esophageal squamous cell carcinoma and its clinical significance. Int J Cancer 122: 325-332, 2008.

15. Bi Q, Tang S, Xia L, et al: Ectopic expression of MiR-125a inhibits the proliferation and metastasis of hepatocellular carcinoma by targeting MMP11 and VEGF. PLoS One 7: e40169, 2012.

16. Higashitsuji $\mathrm{H}$, Higashitsuji $\mathrm{H}$, Itoh $\mathrm{K}$, et al: The oncoprotein gankyrin binds to MDM2/HDM2, enhancing ubiquitylation and degradation of p53. Cancer Cell 8: 75-87, 2005.

17. Lozano G and Zambetti GP: Gankyrin: an intriguing name for a novel regulator of p53 and RB. Cancer Cell 8: 3-4, 2005.

18. Qian YW, Chen Y, Yang W, et al: p28(GANK) prevents degradation of Oct4 and promotes expansion of tumor-initiating cells in hepatocarcinogenesis. Gastroenterology 142: 1547-1558, 2012.

19. Chu YW, Yang PC, Yang SC, et al: Selection of invasive and metastatic subpopulations from a human lung adenocarcinoma cell line. Am J Respir Cell Mol Biol 17: 353-360, 1997.

20. Tie J, Pan Y, Zhao L, et al: MiR-218 inhibits invasion and metastasis of gastric cancer by targeting the Robol receptor. PLoS Genet 6: e1000879, 2010.

21. Bai ZF, Tai Y, Li W, et al: Gankyrin activates IL-8 to promote hepatic metastasis of colorectal cancer. Cancer Res 73: 4548-4558, 2013.

22. Mine H, Sakurai T, Kashida H, et al: Association of gankyrin and stemness factor expression in human colorectal cancer. Dig Dis Sci 58: 2337-2344, 2013.

23. Zhen C, Chen L, Zhao Q, et al: Gankyrin promotes breast cancer cell metastasis by regulating Rac1 activity. Oncogene 32: 3452-3460, 2013. 